\title{
La Internalización de los Costes Medioambientales en el transporte de mercancías por carretera ${ }^{1}$
}

\section{Internalizing Environmental Costs on the road freight transport industry}

\author{
Vera Ferrón VílcheZ ${ }^{2}$ \\ Juan Alberto Aragón Correa ${ }^{2}$ \\ María Dolores Vidal Salazar ${ }^{2}$ \\ Universidad de Granada
}

Recibido el 16 de diciembre de 2009, aceptado el 15 de septiembre de 2010

$\mathrm{N}^{\circ}$ de clasificación JEL: M10; M11; M19

DOI: $10.5295 / \mathrm{cdg} .100197 \mathrm{vf}$

Reseña bibliográfica: FERRÓN, V.; ARAGÓN, J. A. y VIDAL, M. D. (2011): "La Internalización de los Costes Medioambientales en el transporte de mercancías por carretera", Cuadernos de Gestión, Vol 11, nº 1, pp. 117-139, DOI: 10.5295/cdg.100197vf

\section{Resumen:}

La literatura existente señala el potencial de una gestión medioambiental proactiva en la generación de capacidades organizativas relativas a la minimización de costes. Además, durante los últimos años, la internalización de los costes medioambientales de la industria transportista ha sido un importante objetivo en el desarrollo de políticas públicas. Por tanto, este trabajo pretende, en primer lugar, conocer cómo las empresas transportistas pueden afrontar el reto de la creciente importancia adquirida por los denominados costes medioambientales mediante la internalización de los mismos y, en segundo lugar, mostrar cómo las empresas medioambientalmente proactivas estarán capacitadas para afrontar con éxito futuras regulaciones más estrictas en relación a la problemática presentada. Para ello, se ha efectuado un análisis cualitativo basado en una empresa transportista española. La conclusión principal obtenida es que, a pesar de que en la actualidad las empresas transportistas afrontan el reto medioambiental conforme los requerimientos legales van apareciendo, una actitud medioambiental proactiva refuerza un crecimiento empresarial sostenible.

\footnotetext{
${ }^{1}$ Los autores agradecen sobremanera la atención prestada tanto por el equipo directivo como por el personal de Hermanos Aznar S.A., y muy especialmente a D. Antonio Aznar Domínguez, actual gerente de la empresa puesto que sin su colaboración hubiese sido imposible realizar el presente trabajo. Los autores agradecen las valiosas y constructivas sugerencias realizadas por los revisores anónimos de la revista, las cuales han mejorado sustancialmente la calidad académica del presente artículo, y la labor realizada por los editores. Este trabajo ha sido parcialmente cofinanciado por el proyecto con código ECO2009-09241 del Ministerio de Ciencia y Tecnología y el Proyecto de Excelencia con código P06-SEJ-2356 de la Consejería de Innovación, Ciencia y Empresa de la Junta de Andalucía.

${ }^{2}$ Universidad de Granada. Facultad Ciencias Económicas y Empresariales. Campus Cartuja S/N. 18071. Granada (España). Email: vferron@ugr.es; jaragon@ugr.es; lvidal@ugr.es
} 


\begin{abstract}
:
Prior literature noted the potential that advanced environmental management might have in the generation of valuable organizational capabilities regarding costs minimization. More-over, during last years, one of the most important objectives in the development of transport public policies has been the internalization of environmental costs. Thus, this study attempts, firstly, to analyze how transport companies could successfully face the challenge of internalis-ing environmental costs, and second, to highlight how the existence of an environmentally proactive pattern of behaviour might reduce the environmental costs on transport sector. In doing so, a case study in the Spanish industry of road freight transport has been elaborated. Despite nowadays companies face the environmental challenge not beyond legal require-ments, the main conclusion of this study is that an environmentally proactive behaviour re-inforces the sustainable development of companies.
\end{abstract}

\title{
Palabras clave:
}

Costes medioambientales, Proactividad medioambiental, Transporte, Análisis cualitativo.

\section{Keywords:}

Environmental Costs, Environmental Proactivity, Transport, Qualitative Analysis. 


\section{INTRODUCCIÓN}

La actividad económica afecta al medio ambiente tanto por el consumo de materiales y energías como por la generación de residuos y emisiones contaminantes. Este hecho da lugar a unos costes medioambientales que, en algunos casos, son soportados por las empresas (Gibson y Martin, 2004). Consecuentemente, la disminución de estos costes está adquiriendo una creciente importancia en el ámbito empresarial. Además, el Gobierno español aprobó el pasado 27 de noviembre de 2009 el anteproyecto de la denominada Ley de Economía Sostenible, la cual establece reformas normativas para impulsar un crecimiento económico equilibrado y sostenible para con el medio ambiente. Sus tres ejes principales de actuación son la mejora del entorno económico, el aumento de la competitividad de las empresas españolas y la sostenibilidad medioambiental. Por tanto, estas circunstancias ponen de manifiesto la necesidad de una mayor aproximación por parte de las empresas hacia comportamientos medioambientales proactivos, entendiendo dicha proactividad como la tendencia de las organizaciones para iniciar cambios en sus políticas empresariales con antelación, en vez de reaccionar a los eventos a posteriori (Aragón, 1998).

La literatura de gestión medioambiental basada en la teoría de recursos y capacidades (Wernerfelt, 1984; Barney, 1991) ha realizado importantes contribuciones en los últimos años para mostrar el potencial que una gestión medioambiental proactiva puede tener en la generación de capacidades organizativas relativas a la minimización de los costes empresariales (Hart, 1995; Hart y Ahuja, 1996; Nehrt, 1998; Christmann, 2000; Darnall y Edwards, 2006). Así, por ejemplo, Christmann (2000) considera que determinadas prácticas relacionadas con la optimización del proceso productivo de las empresas son esenciales para la implantación de estrategias medioambientales proactivas $\mathrm{y}$, simultáneamente, pueden suponer un ahorro en costes en un plazo relativamente corto. Sin embargo, se ha prestado una atención más escasa al tipo específico de costes medioambientales a los que los directivos deben darle una mayor importancia. Los costes medioambientales suelen delimitarse como aquellos gastos claramente relacionados con el control, prevención y eliminación de la contaminación. No obstante, no existe una definición ampliamente aceptada sobre qué subyace bajo la etiqueta de este tipo de costes y cómo pueden ser internalizados por las empresas. Una razón que puede explicar esta circunstancia es que cada sector industrial cuenta con unos costes medioambientales específicos $\mathrm{y}$, por tanto, resulta complejo delimitar una única definición sobre los mismos.

Para el análisis de la internalización de los costes medioambientales, el presente trabajo se centra en la industria transportista de mercancías por carretera por las particularidades que presenta dicho sector económico. Por un lado, este sector será uno de los más afectados por la implantación de la Ley de Economía Sostenible puesto que uno de los objetivos generales de dicha ley, el de la sostenibilidad medioambiental, señala el establecimiento de una regulación económica para el transporte y la movilidad sostenible como centro de interés. Por ejemplo, el artículo 117 de la citada Ley, relativo a la planificación y gestión eficiente de los servicios de transporte, aboga por "la movilidad sostenible en términos económicos y medioambientales a través de proyectos cuya ejecución produzca una efectiva reducción de las emisiones contaminantes y otros daños al medioambiente (...)". De forma paralela, el ahorro energético y la disminución de gases de efecto invernadero suponen factores críticos dentro del objetivo común de la sostenibilidad económica y medioam- 


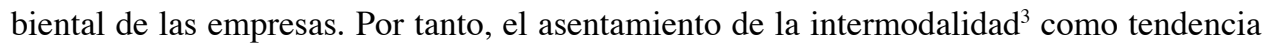
en el transporte de mercancías y los incentivos propuestos para un mayor uso del ferrocarril suponen dos potenciales sustitutos que, tras una futura puesta en marcha de la Ley de Economía Sostenible, amenazan la supervivencia de aquellas empresas transportistas que no afronten desde el principio el reto medioambiental. Por otro lado, el Libro Verde de la Comisión Europea (1995) ha estimado que los costes ocasionados por el sector del transporte de mercancías por carretera con respecto al ruido y la contaminación generada suponen un 0,6\% del PIB comunitario. Durante los últimos años, la estimación e internalización de los costes medioambientales de la industria transportista ha sido un importante objetivo en el desarrollo de políticas públicas a nivel europeo (v.g., Comisión Europea, 1995; 2001; Maibach et al., 2007). Por tanto, el objetivo principal de este trabajo es doble: en primer lugar, analizar cómo las empresas transportistas españolas pueden afrontar el contexto económico y medioambiental planificado sin ver mermada su competitividad, y de forma paralela, poner de manifiesto como un comportamiento medioambiental proactivo puede ayudar a los directivos en su toma de decisiones relativa a la gestión de los costes medioambientales que deben ser internalizados. Para acometer estos objetivos, el presente trabajo ha realizado un análisis cualitativo basándose en una empresa representativa dentro del sector del transporte de mercancías por carretera en España.

Este estudio se divide en cuatro partes fundamentales siguiendo a esta introducción. El segundo apartado recoge una revisión teórica sobre la gestión de los costes medioambientales basada en la teoría de recursos y capacidades. El tercer apartado presenta una aproximación dentro del contexto europeo sobre los costes medioambientales relativos al sector transportista. El cuarto apartado ofrece un análisis cualitativo basado en una empresa representativa del sector del transporte de mercancías por carretera en España. Finalmente, el quinto apartado resume las principales conclusiones, limitaciones y líneas de investigación futura extraídas de la elaboración de este trabajo.

\section{LA GESTIÓN DE LOS COSTES MEDIOAMBIENTALES BAJO LA TEORÍA DE RECURSOS Y CAPACIDADES}

La teoría de recursos y capacidades ofrece un marco de referencia que explica el logro de ventajas competitivas como resultado del desarrollo de capacidades generadoras de valor por parte de las organizaciones (Wernerfelt, 1984; Barney, 1991). Dicha perspectiva teórica argumenta que aquellas empresas que son capaces de acumular recursos y capacidades que son difíciles de obtener, que no son fácilmente imitables por el resto de competidores, que aportan valor a la empresa y que no son sustituibles estarán capacitadas para obtener una ventaja competitiva (Barney, 1991). En relación con el medio ambiente, varios estudios basados en esta teoría han puesto de manifiesto la posibilidad de reducir costes como consecuencia de mejoras en el desarrollo de capacidades proactivas relativas a los objetivos medioambientales de la empresa (Hart, 1995; Christmann, 2000; Darnall

\footnotetext{
${ }^{3}$ La Comisión Europea define la intermodalidad como la característica de un sistema de transportes en virtud de la cual se utilizan de forma integrada al menos dos modos de transporte diferentes (ferroviario, marítimo, por carretera) para completar una cadena de transporte puerta a puerta.
} 
y Edwards, 2006). Por ejemplo, el trabajo seminal de Stuart Hart (1995) argumenta que las estrategias medioambientales dedicadas al desarrollo de actividades centradas en la prevención de la contaminación generan determinadas capacidades organizativas que están en relación con la minimización de los costes empresariales. Específicamente el ahorro en costes mediante la prevención de la contaminación evita el gasto ocasionado por la inversión en determinadas tecnologías relativas al control de la contaminación (Hart, 1995; Sharma y Vredenburg, 1998) y puede incrementar la productividad y eficiencia, ya que una reducción en los residuos se traduce en un mejor uso de los insumos (Hart y Ahuja, 1996). Pero no sólo las actividades dedicadas a la prevención de la contaminación son capaces de disminuir los costes de las empresas. La cuantía de costes puede ser minimizada mediante la reducción (e inclusión eliminación) de sanciones legales como las multas por el incumplimiento de la regulación medioambiental (Sharma y Vredenburg, 1998). Por tanto, todas estas acciones medioambientales relativas al proceso productivo pueden hacer disminuir los costes (Christmann, 2000; Nehrt, 1998) y, simultáneamente permiten que la empresa reduzca sus impactos medioambientales negativos.

En el contexto empresarial actual existe un creciente interés por la minimización de determinados costes derivados del impacto medioambiental de las empresas. Según el estudio de Ditz et al. (1995), los costes medioambientales pueden llegar a alcanzar entre un cinco y un veinte por ciento de los costes totales de la actividad empresarial. No obstante, a pesar de que la relevancia de los costes medioambientales aumenta progresivamente, existe un problema conceptual, al no existir una definición comúnmente aceptada en la literatura que determine qué son costes medioambientales (Gibson y Martin, 2004; UNDSD, 2001). La mayoría de las propuestas desarrolladas internacionalmente a este respecto incluyen bajo la etiqueta de "costes medioambientales" a aquellos costes claramente relacionados con el control o eliminación de la contaminación, tales como los costes para prevenir la generación de emisiones contaminantes o los costes de tratamiento de residuos generados (Savage y Jash, 2004). Consecuentemente, un estudio detallado sobre cuáles son los costes medioambientales que una empresa debe soportar supone el primer paso para minimizarlos y determinar una forma eficiente de reducir potenciales externalidades negativas en relación con el medio ambiente. En otras palabras, un mayor conocimiento sobre los costes medioambientales por parte de los directivos facilitará que las empresas puedan afrontar los mismos de manera efectiva. Los sistemas tradicionales de gestión no tienen suficiente alcance a la hora de analizar los costes medioambientales puesto que ignoran muchos de ellos (y sus potenciales ahorros), lo cual afecta negativamente a las decisiones empresariales. Por tanto, una adecuada gestión de estos costes supondrá no solo mejoras en el rendimiento económico sino también en la disminución de los impactos negativos medioambientales de las empresas. Así, cuanto mayor es el compromiso medioambiental de la empresa, más capacitada estará la misma para disminuir sus costes mediante unos mayores niveles de eficiencia, una mejor gestión de los recursos disponibles o la anticipación a regulaciones medioambientales más restrictivas. Entendiendo la proactividad medioambiental como la tendencia de las empresas para iniciar cambios en sus políticas medioambientales con antelación, en vez de reaccionar a los eventos a posteriori (Aragón, 1998), los directivos de las empresas que cuenten con planteamientos medioambientales proactivos tendrán un mayor interés por anticiparse a la aparición e internalización de los costes medioambientales, y, consecuentemente, estas empresas contarán con un mayor nú- 
mero de recursos y capacidades para afrontar determinadas contingencias que pueden sobrevenir, como ciclos económicos menos rentables, un aumento en el nivel de rivalidad del sector o la entrada en vigor de legislaciones más restrictivas. Por tanto, proponemos que:

\section{PROPOSICIÓN: “Las empresas que siguen comportamientos medioambientales proactivos contarán con un mayor número de recursos y capacidades organizativas para afrontar con éxito la internalización de los costes medioambientales"}

\section{UNA PERSPECTIVA EUROPEA DE LOS COSTES MEDIOAMBIENTALES EN EL SECTOR DEL TRANSPORTE}

Para arrojar luz sobre la proposición de investigación planteada, a lo largo de este apartado, en primer lugar, se presenta cual ha sido la evolución seguida por la industria transportista dentro del ámbito de la Unión Europea y, en segundo lugar, se comentan cuales son las peculiaridades de dicho sector en cuanto a la internalización de los costes medioambientales.

Resulta de especial interés centrar el foco de atención en el sector transportista por tres razones principales. En primer lugar, la actividad transportista está íntimamente conectada con los problemas medioambientales no solo por la generación de contaminación sino también por la problemática de la utilización de insumos no renovables (por ejemplo, petróleo). En segundo lugar, este sector debe cumplir estrictos requerimientos legales tanto en materia medioambiental como en seguridad y riesgos laborales. Los activistas medioambientales suelen centrar sus presiones sociales en este sector económico para reclamar un mayor número de regulaciones medioambientales. Finalmente, y centrándonos en el subsector del transporte de mercancías por carretera, el alto nivel de externalidades negativas generado es uno de los principales objetivos de crítica por parte de organizaciones ecologistas, reguladores públicos y la sociedad en general.

\subsection{El proceso liberalizador del sector transportista en Europa}

Durante las últimas décadas, el sector transportista europeo ha sufrido un importante proceso de liberalización económica. La publicación del Libro Blanco de la Comisión Europea en 1985, en el que se admitía la necesidad tanto del establecimiento de un marco común europeo para los servicios de transporte como de una progresiva liberalización del sector, supuso un punto de inflexión para el transporte europeo. Antes del citado informe, no existía un desarrollo normativo extenso sobre cómo acometer la liberalización del sector transportista en Europa debido a la confrontación entre los grupos que abogaban por dicha liberalización y determinados Estados miembro con una tradición económica menos liberal (Martínez, 1989). Sin embargo, el cambio comenzó en 1983 cuando el Parlamento Europeo acusó a la Comisión de la falta de avances en la puesta en marcha de una política comunitaria en materia de transporte. La sentencia de dicha acusación derivó en la elaboración del Libro Blanco de 1985, el cual trataba la progresiva armonización de los mercados nacionales (De Rus y Campos, 2006). Tras este hito, comenzó la consolidación del proceso liberalizador del sector transportista y, en 1992, se presentó el segundo Libro Blanco de 
la Comisión Europea sobre el transporte comunitario. El principio que sirvió como hilo conductor de este documento fue la apertura del sector transportista, abogando por la consolidación de una política de transporte comunitaria. En la actualidad, el desarrollo de la política de transporte europea está en su última etapa de implantación. El reciente Libro Blanco del sector del transporte (Comisión Europea, 2001) considera que la liberalización del sector ya se ha llevado a cabo y, por tanto, establece nuevos y renovados objetivos para el transporte europeo como el logro de la sostenibilidad medioambiental, el establecimiento de mayores y mejores medidas de seguridad, la incentivación de la intermodalidad, entre otros (De Rus y Campos, 2006). La Ilustración 1 recoge un resumen de cuatro fases del proceso liberalizador de la política europea de transporte hasta la actual situación de dicho sector económico.

Figura 1

Las cuatro fases del proceso liberalizador del transporte europeo



Fuente: De Rus y Campos (2006)

\subsection{Los costes medioambientales y su regulación para el transporte europeo}

De acuerdo con De Rus y Campos (2006), la regulación europea para el sector transportista se basa en tres pilares básicos. En primer lugar, la integración de infraestructuras y servicios, que recoge cómo las empresas dedicadas a la construcción y mantenimiento de infraestructuras (carreteras, autovías, autopistas, puertos, aeropuertos, etc.) operan de manera conjunta con las empresas que usan dichas infraestructuras para ofrecer servicios de transporte. En segundo lugar, los efectos positivos originados por el aumento de redes eficientes de transporte, como el desarrollo del nivel económico de las naciones o la generación de economías de alcance. Finalmente, la especial importancia de los efectos negativos generados por el sector en términos de daños causados y población afectada. Puesto que el principal objetivo del presente trabajo es analizar los costes medioambientales del sector bajo la perspectiva de la gestión de las empresas, nos centraremos en este último fundamento económico, es de- 
cir, el análisis de las externalidades negativas generadas por el sector del transporte europeo. En términos de costes medioambientales, las externalidades son entendidas como aquellos gastos que son generados como consecuencia de impactos medioambientales negativos de la actividad empresarial pero que son pagados o soportados por el conjunto de la sociedad. La literatura previa sobre transporte y medio ambiente ha analizado ampliamente los costes causados por el sector transportista (Mayeres et al., 1996; Eriksen, 2000; Sælensminde, 2004, Quinet, 2004; Haller et al., 2007; Lemp y Kockelman, 2008; Arimura y Iwata, 2008) e incluso varios estudios han realizado clasificaciones de costes externos causados por el transporte. Por ejemplo, Lemp y Kockelman (2008) clasificaron los costes externos medioambientales del transporte en función del tipo de vehículo, mientras que otros estudios centraron su atención en los diferentes costes externos causados por el transporte rodado en las ciudades (Mayeres et al. 1996; Eriksen, 2000; Sælensminde, 2004). Para el presente trabajo se ha utilizado la clasificación de costes medioambientales ofrecida por la Guía de estimación de costes externos del sector del transporte (Maibach et al. 2007) realizada a petición del Directorio General de Transporte y Energía (DG TREN) ${ }^{4}$ de la Comisión Europea por un grupo de investigadores de CE Delft, organización independiente de consultoría e investigación especializada en medio ambiente. Esta guía nace tras la adopción el 27 de marzo de 2006 de la Directiva 1999/62/EC de la Comisión Europea (también conocida como Directiva Eurovignette), en la cual se capacitó a los Estados miembros para imponer peajes en todas las carreteras. Esta guía presenta las mejores prácticas relativas a la estimación de los costes externos del sector transportista en Europa, basándose en diversos informes de expertos e investigadores realizados en el ámbito de la Unión Europea. El documento recoge una clasificación de seis categorías de costes externos del transporte: los costes de congestión del tráfico, los costes de los accidentes, los costes de contaminación del aire, los costes de la contaminación acústica (costes por el ruido generado), los costes relativos al cambio climático y una última categoría que recoge otros costes externos no tenidos en cuenta en los apartados anteriores. En primer lugar, los costes de la congestión del tráfico son los ocasionados por la saturación de las infraestructuras proveniente de la perturbación mutua entre usuarios que compiten por la capacidad limitada del sistema de transporte (Maibach et al. 2007). Estos costes tienen un componente interno (soportado por el usuario) relativo a la pérdida de tiempo y los perjuicios causados al vehículo (depreciación, aumento del gasto en combustible, etc.), pero también tienen un componente externo relacionado con el malestar ocasionado a todos los usuarios en su conjunto y que genera decrementos en el bienestar social. Segundo, los costes de los accidentes son aquellos costes relativos a los accidentes de tráfico que no están cubiertos por las primas de los compañías aseguradoras. Por tanto, el alcance de este tipo de costes no dependerá del nivel del accidente (leve, grave, muy grave) sino del seguro elegido por parte del usuario. Tercero, los costes de contaminación del aire son causados por determinadas emisiones contaminantes como consecuencia de la actividad transportista. Estos costes consisten en costes sanitarios, daños materiales y pérdidas en las cosechas y en los ecosistemas. En cuarto

\footnotetext{
${ }^{4}$ Nótese que desde el 17 de febrero de 2010, el Directorio General de Transporte y Energía (DG TREN) ha sido segregado en dos: el Directorio General de Energía (DG Energy) y el Directorio General de Movilidad y Transporte (DG MOVE).
} 
lugar, los costes del ruido ${ }^{5}$ recogen los costes ocasionados por el malestar y el detrimento de la salud de las personas. Existen dos tipos de impactos negativos como consecuencia del ruido relativo al transporte. Por un lado, el ruido emitido por los vehículos implica un malestar social que restringe o dificulta cualquier actividad de ocio, lo que provoca incomodidades sociales y demás inconvenientes (por ejemplo, depresiones). Por otro lado, el ruido emitido por los vehículos también puede derivar en costes sanitarios relacionados con diversos daños físicos (por ejemplo, los niveles de ruido superiores a 85 decibelios causan pérdidas de audición y, además, la exposición a altos niveles de ruido aumenta el riesgo cardiovascular) y daños psicológicos (como el detrimento de la calidad del sueño). Quinto, los costes relativos al cambio climático o calentamiento global de la tierra hacen referencia a los impactos negativos causados por la actividad transportista como consecuencia principalmente de las emisiones de gases de efecto invernadero. Finalmente, dentro de la categoría "otros costes externos", la Guía incluyen los costes por la contaminación del paisaje, los costes en áreas de especial sensibilidad medioambiental y/o los costes de la dependencia energética. La Tabla 1 muestra de manera simplificada los costes que componen esta categoría en la Guía.

\section{Tabla 1}

\section{Otros costes externos generados por el transporte}

\begin{tabular}{|l|l|}
\hline \multicolumn{1}{|c|}{$\begin{array}{c}\text { Otros costes externos } \\
\text { incluidos }\end{array}$} & \multicolumn{1}{c|}{ ¿Qué incluye cada tipo de costes externos? } \\
\hline $\begin{array}{l}\text { Costes para el entorno } \\
\text { natural y el paisaje }\end{array}$ & $\begin{array}{l}\text { Pérdida del hábitat } \\
\text { Fragmentación del hábitat } \\
\text { Pérdida en la calidad del hábitat }\end{array}$ \\
\hline $\begin{array}{l}\text { Costes por la } \\
\text { contaminación del } \\
\text { terreno y el agua }\end{array}$ & $\begin{array}{l}\text { Emisiones de metales básicos e hidrocarburos aromáticos policíclicos } \\
\text { (PAH) } \\
\text { Amenazas en la flora y fauna } \\
\text { Disminución en la fertilidad del terreno }\end{array}$ \\
\hline $\begin{array}{l}\text { Costes externos en áreas } \\
\text { de especial sensibilidad } \\
\text { medioambiental }\end{array}$ & $\begin{array}{l}\text { Contaminación en áreas de especial sensibilidad medioambiental como } \\
\text { regiones montañosas (Alpes, Pirineos) }\end{array}$ \\
\hline $\begin{array}{l}\text { Costes relativos a los } \\
\text { procesos ascendentes y } \\
\text { descendentes }\end{array}$ & $\begin{array}{l}\text { Impactos medioambientales indirectos como consecuencia de: } \\
\text { - La producción de energía (por ejemplo, combustibles) } \\
\text { - La elaboración, mantenimiento y eliminación de vehículos } \\
\text { - La construcción y mantenimiento de infraestructura para el transporte. }\end{array}$ \\
\hline $\begin{array}{l}\text { Costes adicionales en } \\
\text { áreas urbanas }\end{array}$ & $\begin{array}{l}\text { En las áreas urbanas el tráfico motorizado tiene efectos perniciosos } \\
\text { sobre el tráfico no motorizado (peatones, bicicletas) }\end{array}$ \\
\hline $\begin{array}{l}\text { Costes de la dependen- } \\
\text { cia energética }\end{array}$ & $\begin{array}{l}\text { La distribución desigual de las fuentes energéticas en las diferentes } \\
\text { regiones del mundo hace que existan costes externos del transporte } \\
\text { como consecuencia de la fuerte dependencia con respecto a los países } \\
\text { productores de petróleo (la mayoría organizados mediante el cartel de } \\
\text { la OPEP). }\end{array}$ \\
\hline
\end{tabular}

Fuente: Maibach et al. (2007)

${ }^{5}$ El ruido es definido como el sonido no deseado con duración, intensidad u otra cualidad que causa daños fisiológicos y/o psicológicos a los seres humanos. 
A pesar del extenso y exhaustivo trabajo realizado por los autores de la Guía sobre estimación de costes medioambientales del transporte en Europa, ésta no queda exenta de críticas. Varios investigadores del Instituto de Economía del Transporte de la Universidad de Colonia a petición de la Asociación Europea de Productores de Automóviles (ACEA) realizaron un informe que criticaba diversos aspectos de la Guía (Baum et al., 2008). Estas críticas se basan en que la Guía solo tiene en cuenta los costes externos del transporte, pero no considera las externalidades positivas o beneficios generados por este sector, en que la estimación de costes efectuada no cuantifica las tasas e impuestos que actualmente pagan los transportistas y en que la internalización de costes externos propuesta por la Guía no tiene en cuenta la pérdida de empleos en el sector y los potenciales incrementos inflacionistas que puede acarrear dicha internalización (Baum et al., 2008). Además, Baum et al. (2008) ponen en duda la consideración de determinadas categorías de costes de la Guía como costes externos del transporte. Por ejemplo, esta revisión crítica considera que los costes de congestión del tráfico y, de manera parcial, los costes del ruido son soportados por los usuarios, así como gran parte de los costes de los accidentes son soportados por las empresas aseguradoras. Por tanto, según Baum et al. (2008), los verdaderos costes ocasionados por el transporte que deben ser internalizados por las empresas deben ser los costes de la contaminación del aire, los costes relativos al cambio climático y una porción tanto de los costes del ruido como de los costes por accidentes (Baum et al. 2008). En relación a este tema, el presente trabajo centra la atención en aquellos costes externos directamente relacionados con el medio ambiente, concretamente los costes por la contaminación del aire y los costes relacionados con el cambio climático.

\section{A. Los costes por la contaminación del aire generados por el transporte}

Los costes por la contaminación del aire (CCA) son causados por las emisiones contaminantes de material en partículas (PM10/ PM2.5), óxido de nitrógeno ( $\mathrm{NO}_{x}, \mathrm{NO}_{2}$ ), óxido sulfúrico ( $\mathrm{SO} 2)$, ozono $(\mathrm{O} 3)$ y demás componentes orgánicos volátiles (VOC) como consecuencia de la actividad transportista. Estos costes consisten en costes sanitarios, daños materiales (por ejemplo, daños en las edificaciones), pérdidas en las cosechas y otros costes por daños en los ecosistemas. El estado de la investigación sobre este tipo de costes se encuentra mucho más avanzado que en otros tipos de costes externos como consecuencia principalmente de las estimaciones llevadas a cabo por el modelo ExternE creado por varios proyectos de investigación de la Unión Europea. La Tabla 2 muestra diferentes impactos que relacionados con los CCA.

Para el transporte por carretera, el factor más relevante para estimar los CCA es la cuantía de las emisiones del vehículo, la cual depende tanto de las diferentes características del vehículo empleado (edad, velocidad, tipo de combustible, tecnología de combustión empleada, carga, tamaño) como del patrón de conducción seguido y las peculiaridades geográficas de la carretera. 
Tabla 2

Costes por los impactos contaminantes del aire generados por el transporte

\begin{tabular}{|l|l|}
\hline \multicolumn{1}{|c|}{ Tipos de CCA } & \multicolumn{1}{c|}{ Definición } \\
\hline Costes sanitarios & $\begin{array}{l}\text { Impactos en la salud humana debido a la aspiración de partículas diminutas } \\
\text { (por ejemplo, PM2.5/PM10). }\end{array}$ \\
\hline $\begin{array}{l}\text { Daños materiales } \\
\text { y daños en las } \\
\text { edificaciones }\end{array}$ & $\begin{array}{l}\text { Impactos en edificios y materiales por los contaminantes aéreos. Los dos } \\
\text { efectos de mayor importancia son, en primer lugar, la suciedad de las } \\
\text { fachadas de los edificios por las partículas y el polvo y, en segundo lugar, la } \\
\text { degradación mediante los procesos corrosivos debido a los contaminantes } \\
\text { ácidos del aire, como el NOx y el SO2. }\end{array}$ \\
\hline $\begin{array}{l}\text { Pérdidas en las } \\
\text { cosechas }\end{array}$ & $\begin{array}{l}\text { Tanto las cosechas como los bosques y demás ecosistemas son dañados por } \\
\text { la emisión de ácidos, la exposición al ozono y el SO2 }\end{array}$ \\
\hline $\begin{array}{l}\text { Otros costes por } \\
\text { daños en los } \\
\text { ecosistemas }\end{array}$ & $\begin{array}{l}\text { Impactos in tierra y agua causados por la eutroficación y acidificación }{ }^{6} \text { de- } \\
\text { bido tanto a la emisión de óxidos de nitrógeno como a la contaminación con } \\
\text { metales duros (puesta y retirada de neumáticos). }\end{array}$ \\
\hline
\end{tabular}

Fuente: Maibach et al. (2007)

Los CCA suponen una categoría esencial para el análisis de los costes medioambientales en el sector transportista. Un número considerable de estudios y metodologías han centrado la atención en el análisis de este tipo de costes. A este respecto, los dos proyectos de investigación más importantes en el ámbito europeo son, por un lado, la iniciativa "Desarrollo de una perspectiva europea armonizada para los sistemas de costes del transporte y evaluación de proyectos" desarrollada por el Directorio General de Energía y Transportes de la Comisión Europea y conocida por sus siglas (en inglés) como proyecto HEATCO y, por otro lado, el análisis coste beneficio de la iniciativa “Aire Limpio para Europa” llevada a cabo a instancias de la Comisión Europea en 2001 y reconocido por sus siglas (en inglés) CAFE CBA. Ambos proyectos son los encargados de analizar en términos monetarios los efectos perniciosos de altos niveles de contaminación en el aire debidos a la actividad transportista en el ámbito europeo. A pesar de que estas iniciativas ofrecen como resultado el coste unitario por tonelada de emisión contaminante en el aire, existen diversas diferencias relativas a las metodologías usadas. Por ejemplo, ambos proyectos usan diferentes fuentes y técnicas para evaluar en términos monetarios la toxicidad de las emisiones de PM2.5/PM10; mientras que el proyecto CAFE CBA considera que todas las partículas son igual de agresivas, el proyecto HEATCO diferencia a las partículas atendiendo a su nivel de toxicidad en la combustión. Además, la estimación de CCA del proyecto HEATCO otorga un mayor peso a los daños materiales que el proyecto CAFE CBA. Para paliar los inconvenientes del uso de una u otra metodología en la estimación de los CCA, Maibach

\footnotetext{
${ }^{6}$ La eutroficación o eutrofización consiste en el enriquecimiento de las aguas por nutrientes, lo cual provoca la aparición de algas que consumen gran parte del oxígeno impidiendo que la fauna acuática presente pueda habitar. La eutrofización provoca la pérdida de transparencia del agua (disminuyendo la fotosíntesis por la falta de luz) y aumenta la descomposición de la materia orgánica. Esto hace que disminuya la concentración de oxígeno y provoca la muerte de organismos aeróbicos. Sin oxígeno, aumentan las fermentaciones y consecuentemente se desprenden gases tóxicos como el metano (CH4) y el amoníaco (NH3)
} 
et al. (2007) recomiendan la utilización de una perspectiva combinada de los resultados de ambos proyectos. Concretamente, la iniciativa TREMOVE, promovida por el Directorio General de Medio Ambiente de la Comisión Europea, ofrece un modelo de evaluación de los efectos de diferentes políticas medioambientales en el sector transportista europeo (www.tremove.org). TREMOVE ofrece una estimación de los CCA en función de los kilómetros recorridos por tamaño y clase de vehículo. En el análisis cualitativo realizado en los siguientes apartados de este trabajo se ofrece el cálculo de dicha estimación de CCA.

\section{B. Los costes relacionados con el cambio climático generados por el transporte}

Los costes relativos al cambio climático (CCC) o al calentamiento global de la tierra como consecuencia de la actividad transportista son causados principalmente por las emisiones de gases efecto invernadero $\left(\mathrm{CO}_{2}\right)$, óxido nitroso $\left(\mathrm{N}_{2} \mathrm{O}\right)$ y metano $(\mathrm{CH} 4)$. En una cuantía menor, las emisiones de refrigerantes (hidrofluorocarbonos o HFC) de los sistemas móviles de aire acondicionado también contribuyen al calentamiento global. Los principales factores de coste de los CCC del transporte son el consumo de combustible y el contenido de carbón del combustible. La Tabla 3 muestra varios impactos del calentamiento global que se materializan en este tipo de costes externos.

La estimación de los CCC es una tarea que entraña un alto nivel de complejidad como consecuencia tanto de la valoración de los propios daños (en unidades físicas) como su traducción en términos monetarios. En primer lugar, la valoración de los perjuicios causados por el cambio climático o el calentamiento global tiene una difícil delimitación debido al hecho de que este tipo de daños externos son globales y a largo plazo, por lo que la estimación de su probabilidad de riesgo es difícil de anticipar o de conocer con exactitud. Algunos impactos son ciertos y probados mediante modelos detallados, mientras que otros potenciales impactos (huracanes, grandes inundaciones) no son a menudo tenidos en cuenta debido a la falta de información sobre la relación entre el calentamiento global y dichos impactos. Además, los impactos de carácter secundario (por ejemplo, conflictos regionales) son incluso más difíciles de cuantificar. Llegado a este punto, las preguntas a resolver podrían ser: ¿cuáles son los impactos físicos estimados para el análisis?, ¿se deben analizar los daños causados en una única región o a nivel global?, ¿se deben analizar los impactos diferenciando entre sectores económicos o tomando la economía en su conjunto? Estas cuestiones pueden ser relativamente fáciles de contestar en el caso de otros costes medioambientales puesto que su cuantificación en unidades físicas está claramente definida. Sin embargo, en el caso de los CCC existen numerosos inconvenientes a la hora de valorar los daños, siendo necesario evaluarlos mediante una perspectiva integrada, que incluya tanto los perjuicios causados como la estrategia para evitarlos (Maibach et al., 2007). En segundo lugar, la valoración económica de los CCC suele ser controvertida. Las estimaciones de CCC disponibles sobre emisiones de gases efecto invernadero varían como consecuencia de los problemas en las valoraciones teóricas utilizadas relativas a la equidad, irreversibilidad e incertidumbre del fenómeno analizado. Por ejemplo, ¿cuál debe ser el horizonte temporal para análisis (y su correspondiente delimitación en años)? En caso de utilizar una evaluación a largo plazo, ¿cuál debe ser la tasa de descuento?; ¿cada país o región incluida en el análisis deben tener diferentes pesos específicos en función a su nivel de emisiones?; ¿cuál debe ser ese peso? 
Tabla 3

Costes relativos al cambio climático generados por el transporte

\begin{tabular}{|l|l|}
\hline \multicolumn{1}{|c|}{ Tipo de daño } & \multicolumn{1}{c|}{ Definición } \\
\hline $\begin{array}{l}\text { Aumento del nivel } \\
\text { del mar }\end{array}$ & $\begin{array}{l}\text { El aumento del nivel del mar conlleva aumentos en costes para prevenir } \\
\text { este fenómeno o, de lo contrario, pérdida de las zonas húmedas. }\end{array}$ \\
\hline $\begin{array}{l}\text { Impactos del uso } \\
\text { abusivo de energía }\end{array}$ & $\begin{array}{l}\text { El uso abusivo de energía dependerá de la temperatura promedio y su } \\
\text { variabilidad. El cambio climático supone un aumento generalizado de las } \\
\text { temperaturas. El ahorro en las necesidades de calefacción en invierno se } \\
\text { ve superado por el aumento en la demanda de refrigeradores en verano. }\end{array}$ \\
\hline Impactos agrícolas & $\begin{array}{l}\text { Los impactos en la agricultura dependen de los cambios regionales tanto } \\
\text { en la temperatura y las precipitaciones como en los niveles de dióxido } \\
\text { de carbono en la atmósfera (y la fertilización). Los impactos clave en las } \\
\text { cosechas se aprecian en los cambios de las áreas de cultivo y los campos. }\end{array}$ \\
\hline $\begin{array}{l}\text { Impactos en el } \\
\text { abastecimiento de } \\
\text { agua }\end{array}$ & $\begin{array}{l}\text { Los impactos en el abastecimiento de agua dependen de los cambios en las } \\
\text { tasas de precipitación y la evapotranspiración y los cambios en la demanda } \\
\text { de agua (ecosistemas, variabilidad en los niveles de humedad, etc.) }\end{array}$ \\
\hline Impactos en la salud & Los impactos sanitarios como consecuencia del cambio en las temperaturas. \\
\hline $\begin{array}{l}\text { Impactos en los } \\
\text { ecosistemas y la } \\
\text { biodiversidad }\end{array}$ & $\begin{array}{l}\text { La productividad ecológica y la biodiversidad son alteradas por el cam- } \\
\text { bio climático y el aumento en el nivel del mar, aumentando el peligro de } \\
\text { extinción de varias especies. }\end{array}$ \\
\hline $\begin{array}{l}\text { Eventos extremos } \\
\text { meteorológicos }\end{array}$ & $\begin{array}{l}\text { Aumentos en los eventos meteorológicos extremos, como olas de calor, } \\
\text { tormentas, sequías, ciclones tropicales, etc. }\end{array}$ \\
\hline $\begin{array}{l}\text { Otros sucesos } \\
\text { importantes }\end{array}$ & $\begin{array}{l}\text { Deshielo del Antártida Oeste. } \\
\text { Deshielos en Groenlandia. } \\
\text { Desniveles de metano. } \\
\text { Inestabilidad y/o colapso en el Amazonas. } \\
\text { Cambios en la circulación termohalina2. } \\
\text { Transformaciones en los monzones indios. } \\
\text { Cambios en la estabilidad de la vegetación sahariana. } \\
\text { Cambios en el albedo tibetano. } \\
\text { Aumentos en las oscilaciones sureñas de "El Niño" (ENSO) } \\
\text { Reducción de la capacidad de los depósitos de carbón } \\
\text { Otros eventos. }\end{array}$ \\
\hline
\end{tabular}

Fuente: Watkiss (2005)

En relación a la valoración económica de los CCC, el debate más controvertido es el que existe entre los estudios que utilizan para la estimación de los CCC la perspectiva del daño causado frente a la perspectiva que aboga por evitar o mitigar la aparición de los CCC. La primera metodología comentada cuantifica en unidades monetarias el perjuicio ya realizado (irreversible). El proyecto denominado "Los Costes Sociales del Carbono" desarrollado por la empresa de consultoría medioambiental "AEA Technology" (http://www. aeat.co.uk) y el Instituto del Medio Ambiente de Estocolmo (http://sei-international.org)

${ }^{7}$ La circulación termohalina se refiere a la que conecta el conjunto de las masas de agua oceánicas. 
han realizado recientemente una evaluación sobre los CCC basándose en esta metodología. Por el contrario, la segunda perspectiva evalúa los costes de evitar las futuras emisiones de $\mathrm{CO}_{2}$, determinando cuál será la opción de menor coste para alcanzar una reducción en el nivel de emisiones de gases de efecto invernadero. Para ello, se puede tomar como referencia el límite impuesto por una política gubernamental (de nivel nacional, de nivel europeo, e incluso de nivel global como el Protocolo de Kyoto). Esta metodología ha sido empleada y recomendada por diversos estudios y proyectos de investigación como, por ejemplo, UNITE, ExternE y el Informe Stern del 2006.

Puesto que, a pesar de sus evidentes diferencias de cálculo y estimación, ambos métodos cuentan con sus ventajas e inconvenientes, Maibach et al. (2007) recomiendan la utilización simultánea de dichas metodologías para la estimación de los CCC. Concretamente, para la estimación a corto plazo, el estudio de Maibach et al. (2007) usa como base la metodología que aboga por evitar la aparición de los CCC, mientras que para el largo plazo utiliza la metodología del perjuicio causado por la emisión de gases de efecto invernadero. Estos autores consideran que, dadas las circunstancias comentadas, la decisión sobre el valor específico que debe tener la estimación de los CCC para la internalización de los mismos por parte de las empresas está altamente influenciada por las decisiones políticas finalmente tomadas, más que por los resultados obtenidos en la investigación al respecto. Así, los autores siguen los valores ofrecidos por el proyecto TREMOVE, los cuales muestran una estimación de los CCC atendiendo a los kilómetros recorridos por tipo y clase de vehículo. En los siguientes apartados se ofrece el cálculo de dicha estimación de CCC.

\section{EL TRANSPORTE DE MERCANCÍAS POR CARRETERA EN ESPAÑA.}

De las agrupaciones económicas del sector servicios en España para el año 2007, el transporte supuso un $8,14 \%$ del volumen total de la cifra de negocio, ocupando el cuarto lugar del total del sector de servicios de mercado no financieros (EAS, 2008). De los cuatro subsectores que conforman el grupo de los servicios vinculados al transporte, el de transporte de mercancías es el que genera la mayor cifra de negocios. Por ejemplo, para el año 2006, más de la mitad de las empresas de la actividad transportista correspondieron al transporte de mercancías $(61,1 \%$ del total), fue el sector que generó el mayor volumen de negocio $(39,5 \%)$ y el que ocupó a la mayor parte del personal empleado en este sector $(42,3 \%)$ (EAS, 2008). Además, de todos los medios de transporte existentes, el transporte de mercancías por carretera supone más de tres cuartas partes de la carga total transportada (EAS, 2008).

\subsection{La regulación española para el transporte de mercancías por carretera.}

El órgano competente a nivel nacional encargado de velar por la competitividad y eficiencia del sector objeto de estudio es la Dirección General de Transportes por Carretera que forma parte del Ministerio de Fomento. Este organismo aprobó en 2001, con un horizonte temporal de cinco años, el "Plan Estratégico para el transporte de mercancías por carretera" (PETRA). El objetivo principal de este plan estratégico es construir un marco 
de referencia en el que se delimiten "las actuaciones conjuntas de todo el sector con el fin último de alcanzar su modernización y, con ello, un posicionamiento competitivo”. En la actualidad, PETRA se encuentra en su segunda fase de implantación, que abarca desde 2007 hasta 2012 y que se le denomina PETRA II puesto que supone una continuación de lo acordado en el plan inicial. En materia medioambiental, PETRA establece como uno de sus pilares básicos la protección del entorno natural en el que se desarrolla la actividad del sector. La Tabla 4 muestra un resumen de los objetivos promulgados por PETRA en relación con sus tres programas de medidas.

Tabla 4

\section{Las medidas medioambientales de PETRA y sus objetivos}

\begin{tabular}{|l|l|}
\hline \multicolumn{1}{|c|}{ Medidas PETRA } & \multicolumn{1}{c|}{ Objetivo de la medida } \\
\hline $\begin{array}{l}\text { Fomento de la inversión en vehículos } \\
\text { ecológicos. }\end{array}$ & $\begin{array}{l}\text { Favorecer y fomentar las inversiones en material móvil } \\
\text { que no perjudique el medio ambiente, de forma que los } \\
\text { costes externos del transporte queden minimizados en } \\
\text { origen }\end{array}$ \\
\hline $\begin{array}{l}\text { Promoción de la seguridad y la iden- } \\
\text { tificación pública de la adaptación } \\
\text { medioambiental }\end{array}$ & $\begin{array}{l}\text { Promover la comparación entre diferentes empresas en } \\
\text { relación con los logros alcanzados en materia medioam- } \\
\text { biental y de seguridad vial, como forma de fomentar la } \\
\text { adopción de "mejores prácticas" }\end{array}$ \\
\hline $\begin{array}{l}\text { Establecimiento de restricciones para } \\
\text { vehículos no adaptados a los requeri- } \\
\text { mientos medioambientales }\end{array}$ & $\begin{array}{l}\text { Favorecer la rápida adopción de medidas ecológicas que } \\
\text { permitan reducir los costes externos en origen }\end{array}$ \\
\hline
\end{tabular}

No obstante, es importante mencionar que, en general, las medidas medioambientales propuestas en PETRA gozan de una importancia media. En otras palabras, mientras que la seguridad, la formación y la calidad en el sector transportista implican líneas de actuación de importancia vital y prioritaria para la mejora de la competividad y la eficiencia, los planes PETRA consideran que las medidas en materia medioambiental deben ser implementadas con una perspectiva temporal de medio plazo.

\subsection{Análisis cualitativo: la empresa transportista Hermanos Aznar S.A.}

\section{A. Metodología}

Con idea de conocer qué sucede en el ámbito práctico en relación con el tema planteado, se ha utilizado la metodología cualitativa del estudio del caso, usando distintas fuentes de información. En primer lugar, como se ha puesto de manifiesto en los apartados anteriores, se han analizado diversas publicaciones y los resultados de varios proyectos de investigación para contextualizar el sector y su problemática en torno a la temática medioambiental (v.g., Maibach et al., 2007; Baum et al., 2008). En segundo lugar, se ha recurrido al Boletín 
Oficial del Registro Mercantil (BORME) con objeto de identificar información objetiva de carácter financiero sobre la empresa objeto de análisis (cifra de negocio, número de empleados, director general, etc.) con idea de comprobar la representatividad de la misma con respecto a la media del sector. En tercer lugar, se mantuvieron dos entrevistas personales con el gerente de la empresa, D. Antonio Aznar Domínguez. Durante la primera entrevista, los investigadores centraron la atención en dos aspectos fundamentales: de manera general, conocer los planteamientos organizativos de la empresa y de manera específica, conocer los planteamientos gerenciales en materia medioambiental. En la segunda entrevista, el foco de interés se centró en conocer cómo afectaría una futura internalización de los costes medioambientales a la marcha diaria de la empresa. En este punto, los investigadores y el gerente comentaron las siguientes preguntas: ¿cuál sería el posible coste a soportar?, ¿qué parte del mismo y cómo se repercutiría al cliente final?, ¿cómo podría evitarse o, al menos, minimizarse? Finalmente, se visitaron las instalaciones de la empresa, y se mantuvieron conversaciones informales con varios de los empleados de la empresa al objeto de contrastar la información aportada.

\section{B. Datos generales de la empresa objeto de análisis.}

La orientación de negocio familiar y estructura típica de esta $\mathrm{PYME}^{8}$ suponen un buen representante del promedio de empresas participantes en el tejido empresarial del sector transportista español. Hermanos Aznar es una empresa que ofrece servicios de transporte de mercancías por carretera. Está localizada en Vera, población costera de la provincia de Almería. Vera es un importante centro de envío de transportes de mercancía por carretera como consecuencia de su situación geográfica integrada en una de las zonas con mayor nivel de exportación agrícola hacia otros países de la Unión Europea. En la actualidad Hermanos Aznar cuenta con una plantilla de 60 empleados, una flota de 57 vehículos y un volumen de negocio ${ }^{9}$ cifrado en más de 3,5 millones de euros para el ejercicio económico de 2007. La empresa está especializada en el transporte internacional de productos agrícolas y goza de relaciones estables con agentes comerciales ubicados en las principales ciudades centroeuropeas en las que se desarrolla la mayor parte del comercio comunitario de este tipo de productos.

\section{Estrategia medioambiental de la empresa}

En lo que se refiere a materia medioambiental, al igual que el resto de competidores del sector, Hermanos Aznar se ve sometida a una estricta regulación que afecta a diferentes áreas de la empresa. Los tres principales focos de atención que ya están siendo atendidos por la empresa desde el punto de vista de la internalización de los costes medioambientales son: el mayor coste soportado como consecuencia de la inversión en vehículos ecológicos

\footnotetext{
${ }^{8}$ Acrónimo utilizado para hacer mención a las pequeñas y medianas empresas.

${ }^{9}$ Según la información contenida en BORME, el resultado de la empresa para el ejercicio económico del 2007 fue de $79.969,80$ euros.
} 
(tipo Euro IV y V), el pago de tasas e impuestos medioambientales en autopistas, combustible y neumáticos, y el coste de la gestión de los residuos. En primer lugar, en lo referente a la inversión de vehículos ecológicos, la legislación actual española obliga a la adquisición de vehículos no contaminantes, por lo que la transformación "verde" de los vehículos se produce conforme la empresa va renovando progresivamente su flota. Hermanos Aznar cuenta con una flota de 57 camiones, de los cuales 13 son del tipo Euro II, 21 son del tipo Euro III y 23 son del tipo Euro IV. Esta circunstancia supone que aproximadamente un $40 \%$ de la flota actual de la empresa corresponde a vehículos ecológicos. En segundo lugar, el pago de tasas e impuestos medioambientales en autopistas y combustible supone un coste medioambiental ya internalizado por la empresa. Por un lado, cuanto mayor es el poder contaminante del vehículo, mayor es el pago que los operadores deben realizar por el uso de las infraestructuras. El gerente comentó que esta medida supone un gasto considerable sobre todo en las autopistas alemanas porque en dicho país es donde se paga el mayor precio por kilómetro recorrido. Por otro lado, la utilización de un componente químico reductor de emisiones al aire (la urea) en el combustible supone un incremento en el coste final del servicio. Concretamente, un camión puede gastar aproximadamente 1 litro del producto Ad-Blue (urea) por cada cien kilómetros y tiene un precio cercano a 0,50 euros por litro. Los vehículos tipo Euro IV y Euro V requieren dicho componente. En tercer lugar, el coste soportado por la gestión de los residuos es la actividad de la cadena de valor de la empresa que más preocupa a la Dirección. Los residuos y desechos producidos en los talleres (filtros, aerosoles, trapos sucios, etc.) deben ser recogidos por empresas dedicadas exclusivamente a la retirada y tratamiento de los mismos, pero dichos servicios no son gratuitos en todos los casos. Dicho servicio es gratuito solo en el caso de la retirada del aceite usado y las baterías, pero tanto las infraestructuras necesarias como las medidas a seguir para ello son "terriblemente estrictas", según comenta el gerente. Por tanto, la gestión de los residuos y desechos supone un coste medioambiental que la empresa soporta actualmente para cumplir los requisitos de la regulación medioambiental.

Analizando el comportamiento medioambiental actual de Hermanos Aznar se observa la existencia de un planteamiento con respecto a sus costes medioambientales que lleva a priorizar el seguimiento de aquellos que van apareciendo conforme las restricciones de la legislación medioambiental avanzan en número y grado. La empresa trata de cumplir estrictamente sus obligaciones medioambientales pero sin traspasar los límites impuestos por la regulación.

No obstante, debido al interés mostrado por la internalización de los costes medioambientales del sector transportista por parte de los reguladores públicos tanto a nivel europeo (entrada en vigor de un decreto que obligase a internalizar un mayor número de costes medioambientales del transporte) como a nivel nacional (entrada en vigor de la Ley de Economía Sostenible), Hermanos Aznar podría enfrentarse en un periodo de tiempo relativamente corto (de 2 a 5 años) a la obligación de internalizar determinados costes medioambientales que hasta ahora no habían sido tenidos en cuenta. A continuación, atendiendo a las estimaciones de costes medioambientales propuestas por Maibach et al. (2007), se calculan los costes medioambientales que la empresa debería soportar en su situación actual.

En relación a los CCA, la estimación de estos costes ha sido cuantificada por el proyecto TREMOVE (Maibach et al., 2007) en base a las toneladas de emisión por kilómetro/ 
vehículo recorrido, diferenciando por tipo de vehículo (normas EURO), su tamaño (toneladas) y los diferentes modos (por carretera, aéreo, marítimo). La Tabla 5 muestra la estimación de CCA que debería internalizar Hermanos Aznar dada su flota actual.

Tabla 5

Estimación de los CCA actuales para Hermanos Aznar

\begin{tabular}{|l|c|c|c|}
\hline \multicolumn{1}{|c|}{ Tipo de vehículo } & $\begin{array}{c}\text { Promedio en cts. de } € \text { por } \\
\text { vehículo/km recorrido }\end{array}$ & $\begin{array}{c}\mathrm{N}^{\mathrm{o}} \text { camiones y promedio de } \\
\text { kms recorridos }\end{array}$ & Total \\
\hline $\begin{array}{l}\text { Camión de }+32 \mathrm{t} \\
\text { Tipo Euro II }\end{array}$ & 9,8 & $\begin{array}{c}13 \text { camiones. } \\
160.000 \mathrm{kms} / \mathrm{año}\end{array}$ & $203.840 €$ \\
\hline $\begin{array}{l}\text { Camión de }+32 \mathrm{t} \\
\text { Tipo Euro III }\end{array}$ & 7,7 & $\begin{array}{c}21 \text { camiones } \\
160.000 \mathrm{kms} / \mathrm{año}\end{array}$ & $258.720 €$ \\
\hline $\begin{array}{l}\text { Camión de }+32 \mathrm{t} \\
\text { Tipo Euro IV }\end{array}$ & 4,6 & $\begin{array}{c}23 \mathrm{camiones} \\
160.000 \mathrm{kms} / \mathrm{año}\end{array}$ & $169.280 €$ \\
\hline \multicolumn{2}{|l|}{ Costes Totales por contaminación del aire $(57$ camiones $)$} & $631.840 €$ \\
\hline
\end{tabular}

Fuente: TREMOVE. Alemania fue la base de evaluación. (Maibach et al. 2007)

Siguiendo la propuesta de costes elaborada por la Guía sobre estimación de los costes externos del transporte (Maibach et al., 2007), Hermanos Aznar tendría que soportar con su flota actual unos CCA totales de 631.840 euros. Sin embargo, si su flota actual estuviera compuesta sólo por vehículos ecológicos (del tipo Euro V) esta cantidad se reduciría hasta los 255.360 euros (con un CCA promedio de 2,8 céntimos de euro por vehículo/km).

En relación con los CCC, la estimación de estos costes ha sido cuantificada por el proyecto TREMOVE (Maibach et al., 2007) en base a las toneladas de $\mathrm{CO} 2$ emitidas por kilómetro/vehículo recorrido, diferenciando por tipo de vehículo (normas EURO), su tamaño (toneladas) y los diferentes modos (por carretera, aéreo, marítimo). La Tabla 6 muestra la estimación de CCC que debería internalizar Hermanos Aznar dada su flota actual.

Tabla 6

Estimación de los CCC actuales para Hermanos Aznar

\begin{tabular}{|l|c|c|c|}
\hline \multicolumn{1}{|c|}{ Tipo de vehículo } & $\begin{array}{c}\text { Promedio en cts. de } € \text { por } \\
\text { vehículo/km recorrido }\end{array}$ & $\begin{array}{c}\mathrm{N}^{\mathrm{o}} \text { camiones y promedio de } \\
\text { kms recorridos }\end{array}$ & Total \\
\hline $\begin{array}{l}\text { Camión de }+32 \mathrm{t} \\
\text { Tipo Euro II }\end{array}$ & 2 & $\begin{array}{c}13 \text { camiones } \\
160.000 \mathrm{kms} / \mathrm{año}\end{array}$ & $41.600 €$ \\
\hline $\begin{array}{l}\text { Camión de }+32 \mathrm{t} \\
\text { Tipo Euro III }\end{array}$ & 2 & $\begin{array}{c}21 \mathrm{camiones} \\
160.000 \mathrm{kms} / \mathrm{año}\end{array}$ & $67.200 €$ \\
\hline $\begin{array}{l}\text { Camión de }+32 \mathrm{t} \\
\text { Tipo Euro IV }\end{array}$ & 1,9 & $\begin{array}{c}23 \mathrm{camiones} \\
160.000 \mathrm{kms} / \mathrm{año}\end{array}$ & $69.920 €$ \\
\hline Costes Totales por contaminación del aire $(57$ camiones $)$ & $178.720 €$ \\
\hline
\end{tabular}

Fuente: TREMOVE. Alemania fue la base de evaluación. (Maibach et al. 2007) 
Según los valores obtenidos por la Guía (Maibach et al., 2007), la estimación de CCC que Hermanos Aznar debería soportar con su flota actual sería de 178.720 euros. Sin embargo, si su flota actual estuviera compuesta sólo por vehículos ecológicos (del tipo Euro V) esta cantidad se reduciría hasta los 173.280 euros (con un CCC promedio de 1,9 céntimos de euro por vehículo/km).

\subsection{Resultados del análisis cualitativo.}

Estos resultados muestran que la internalización de los costes medioambientales puede llegar a afectar fuertemente a la gestión y a la supervivencia de las empresas transportistas españolas, de tal forma que cuanto más capaces sean estas de minimizar los costes medioambientales mediante una mayor proactividad medioambiental, mayores serán sus posibilidades de supervivencia y/o logro de mejoras en la rentabilidad futura. Por ejemplo, ¿cómo afectaría la internalización de los costes medioambientales del transporte atendiendo al principio "el que contamina, paga"? Atendiendo a las estimaciones de costes medioambientales propuestas por Maibach et al. (2007), si Hermanos Aznar repercutiese los costes medioambientales en el precio final del servicio y considerando un porte promedio (sólo ida) de 2.250 kilómetros, que es la distancia entre Vera (Almería) y Ámsterdam (Holanda) utilizando un vehículo tipo Euro II, la empresa tendría que incrementar el precio de su servicio en 265,5 euros, siendo 220,5 euros en concepto de CCA y 45 euros en concepto de CCC. Sin embargo, si este servicio promedio se prestase utilizando un vehículo tipo Euro IV, la empresa tendría que incrementar el precio del porte en 146,25 euros, es decir, 119,25 euros menos que si se utiliza un vehículo no ecológico, siendo 103,5 euros en concepto de CCA y 42,75 euros en concepto de CCC.

Por tanto, los resultados del análisis cualitativo confirman la proposición de investigación que consideraba que las empresas que siguen comportamientos medioambientales proactivos estarán en disposición de contar con un mayor número de recursos y capacidades organizativas para afrontar con éxito la internalización de los costes medioambientales.

\section{DISCUSIÓN: CONCLUSIONES, IMPLICACIONES, LIMITACIONES Y FU- TURAS LÍNEAS}

\subsection{Conclusiones}

El objetivo principal de este trabajo es doble: en primer lugar, conocer cómo las empresas transportistas pueden afrontar el reto de la creciente importancia adquirida por los denominados costes medioambientales mediante la internalización de los mismos y, de forma paralela, en relación con la teoría de recursos y capacidades, nuestro estudio propone que aquellas empresas del sector que se anticipen a la aparición de este tipo de costes mediante un comportamiento medioambiental proactivo estarán capacitadas para afrontar con éxito la entrada en vigor de futuras regulaciones más estrictas. Para ello, se ha efectuado un estudio cualitativo en el sector del transporte de mercancías por carretera en España. Las principales conclusiones que se pueden destacar del análisis efectuado son dos. En primer lugar, un pa- 
trón de funcionamiento generalizado dentro del sector transportista es la reactividad medioambiental ya que muchas de las empresas del sector se limitan a cumplir de forma rigurosa la normativa medioambiental vigente, pero sin traspasar estos límites (Roome, 1992). No obstante, el análisis cualitativo efectuado pone de manifiesto cómo un comportamiento medioambiental proactivo puede implicar un ahorro en costes puesto que, comparando la estimación de costes medioambientales que tendría que soportar Hermanos Aznar con su actual flota (compuesta tanto de vehículos ecológicos como de no ecológicos) con la estimación de costes medioambientales que tendría que soportar con una flota compuesta solo por vehículos ecológicos, la empresa reduciría notablemente sus costes medioambientales y, por tanto, estaría capacitada para ofrecer sus servicios a precios competitivos. Puesto que la actual legislación española tiene un carácter coercitivo en lo relativo a la conversión ecológica de las flotas, es decir, las inversiones en vehículos nuevos deben ser del tipo Euro IV y V, los directivos de las empresas transportistas que, en la actualidad, inviertan en vehículos ecológicos, consuman las materias primas y energías menos contaminantes, optimicen sus procesos productivos siguiendo el principio de la ecoeficiencia y realicen provisiones dedicadas a la anticipación de potenciales impactos medioambientales negativos que pueda causar su empresa contarán con determinados recursos y capacidades organizativas que les ayudarán a afrontar el reto medioambiental y, simultáneamente, verán reducidos el montante de costes medioambientales que deberán soportar. En segundo lugar, a pesar que los reguladores públicos a nivel europeo tienen un interés manifiesto por la internalización de las externalidades negativas medioambientales causadas por el transporte, las instituciones españolas responsables de este sector consideran que los objetivos relacionados con las mejoras del medio ambiente juegan un papel importante, pero secundario, y así queda reflejado en el contenido de los planes PETRA, los cuales dan una "importancia media, de desarrollo a medio plazo" a las actuaciones medioambientales de la industria. Por tanto, puesto que actualmente la problemática medioambiental queda relegada a una prioridad secundaria en el ámbito institucional español, las empresas del sector no tienen la obligación de enfocar sus esfuerzos a la lucha contra el deterioro del medio ambiente más allá de los límites a los que la legislación obliga. Este hecho coincide con los resultados del estudio de Haller et al. (2007), los cuales argumentan que "el déficit o el retraso de la asistencia y la promoción del nivel federal limita severamente las perspectivas para la conversión voluntaria de flotas" (Haller et al., 2007, p. 12). Sin embargo, se prevé que esta circunstancia cambie en un futuro cercano como consecuencia de la implantación de las medidas recogidas en la Ley de Economía Sostenible, ya que esta nueva normativa aboga por una mayor aproximación a un sistema económico medioambientalmente sostenible y, en relación con el sector transportista, un incremento en el uso de servicios de transporte alternativos (como el ferrocarril), que supongan un mayor ahorro energético y menores impactos medioambientales negativos. Por tanto, serán las empresas transportistas con planteamientos medioambientales proactivos las que sobrevivan a este nuevo panorama económico.

\subsection{Implicaciones para la gestión y los reguladores públicos.}

Este trabajo contiene implicaciones importantes tanto para los directivos como para los reguladores públicos. Por un lado, en relación a la gestión, los resultados de análi- 
sis cualitativo efectuado revelan que, para algunos directivos del sector transportista, el reto medioambiental parece entenderse como un incremento en sus costes operativos que hace disminuir los beneficios empresariales. Sin embargo, esta circunstancia difiere de las acontecidas en otros sectores económicos, los cuales han sido testigos de cómo la posición competitiva de sus empresas ha mejorado mediante la diferenciación ecológica de sus productos o servicios, como en el caso de la manufactura de productos perecederos. Por tanto, nuestro trabajo contribuye a la mejora en la gestión de las empresas ya que resalta los potenciales beneficios que conlleva una gestión medioambiental proactiva. Por otro lado, en relación con los reguladores públicos, el análisis del contenido de PETRA pone de manifiesto que los planes estratégicos proyectados para la industria española del transporte de mercancías por carretera aún se encuentra en una etapa incipiente en lo que al desarrollo de actuaciones medioambientales se refiere. Es posible que por este motivo las empresas españolas hagan frente a las necesidades organizativas medioambientales conforme los requerimientos legales van aumentando en número y alcance. Por tanto, este trabajo pone de manifiesto la importancia de otorgar desde el ámbito institucional un papel más trascendental a los efectos perniciosos de la actividad económica para con el medio ambiente, ya que en aquellos sectores medioambientalmente reactivos es necesaria la implantación de determinadas medidas coercitivas para su avance en materia medioambiental.

\subsection{Limitaciones y Futuras Líneas de Investigación.}

Existen tres limitaciones principales en el presente estudio. En primer lugar, la metodología seguida implica un análisis cualitativo de las circunstancias que concurren en una empresa específica, pero no goza de los beneficios del análisis cuantitativo, como puede ser la extrapolación de los resultados a otras empresas y/o sectores. Basándose en estudios cuantitativos ya realizados (v.g., Maibach et al., 2007; Friedrich y Bikel, 2001), futuros trabajos podrán abordar el tema objeto de estudio usando tanto bases de datos con un amplio número de casos como técnicas estadísticas de regresión que permitan corroborar la proposición de investigación planteada de manera cuantitativa en otros sectores, países y/o situaciones empresariales diferentes. En segundo lugar, para obtener una estimación de los costes medioambientales que debería soportar una empresa transportista se ha seguido la propuesta del proyecto TREMOVE (Maibach et al., 2007) para los CCA y los CCC. No obstante, la Guía sobre estimación de los costes medioambientales del transporte ofrece multiplicidad de metodologías de cálculo para la estimación no solo de los CCA y los CCC, sino también para el resto de costes externos del transporte, así como una extensa revisión sobre los estudios e investigaciones llevados a cabo al respecto. Por tanto, otros trabajos podrán obtener estimaciones de costes medioambientales diferentes a los aquí mostrados. Finalmente, el enfoque realizado para el sector transportista limita la aplicabilidad práctica de los resultados obtenidos para otros sectores económicos. Futuras investigaciones serán las encargadas de contrastar si el interés por la internacionalización de los costes medioambientales está positivamente relacionado con la proactividad medioambiental en otras empresas, sectores e incluso países.

Además, estudios futuros podrían intentar corroborar de forma agregada si los mayores volúmenes de ciertos costes medioambientales están correlacionados con otras caracterís- 
ticas de la estrategia de negocio de la empresa. Por ejemplo, cabría plantearse si una mayor orientación innovadora podría suponer una reducción de este tipo de costes, o cuál es el grado de congruencia entre el hecho de perseguir una estrategia de liderazgo en costes (a nivel de unidad de negocio) y la implantación de estrategias medioambientales proactivas. La importancia de la problemática medioambiental y las restricciones a los avances en gestión medioambiental ligados a las consecuencias en costes llevan a poner de manifiesto que este tipo de temas obtendrán un mayor interés analítico en el futuro.

\section{REFERENCIAS BIBLIOGRÁFICAS}

ARAGÓN, J. A. (1998): "Strategic proactivity and firm approach to the natural environment". Academy of Management Journal, Vol. 41, No.5. pp. 556-567

ARIMURA, T.H. Y IWATA, K. (2008): "Economics analysis on motor-vehicle type regulation: policy evaluation of NOx-PM Law". Environmental Science, Vol. 21, pp. 103-114.

BARNEY, J. B. (1991): "Firm resources and sustained competitive advantage". Journal of Management, Vol. 17, No. 1, pp. 99-120

BAUM, H., GEISSLER, T., SCHNEIDER, J. Y BÜHNE, J.A. (2008): External Costs in the Transport Sector: A Critical Review of the EC-Internalisation Policy. Colonia, Institute for Transport Economics.

CHRISTMANN, P. (2000): "Effects of Best Practices of Environmental Management on Cost Advantage: the Role of Complementary Assets". Academy of Management Journal, Vol. 43, pp. 663-680 Comisión Europea. (1985): Completing the Internal Market. Libro Blanco COM(1985) 310.

COMISIÓN EUROPEA. (1992): The development of the future common transport policy. Libro Blanco COM-92 492.

COMISIÓN EUROPEA. (1995): Towards Fair and Efficient Pricing in Transport Policy: Policy Options for Internalising the External Cost of Transport in the European Union. Libro Verde COM-95 691.

COMISIÓN EUROPEA. (1998): Fair payment for infrastructure use: A phased approach to a common transport infrastructure charging framework in the European Union. Libro Blanco COM98466.

COMISIÓN EUROPEA. (2001): European transport policy for 2010: Time to decide. Libro Blanco COM-2001 370.

DARNALL, N. Y EDWARDS, D. (2006): "Predicting the cost of environmental management system adoption: the role of capabilities, resources and ownership structure". Strategic Management Journal, Vol. 27, pp. 301-320.

DE RUS, G. Y CAMPOS, J. (2006): "Los fundamentos económicos de la política de transporte europea: un análisis crítico". La política de transporte europea: el papel del análisis económico. Bilbao, Fundación BBVA.

DITZ, D., RANGANATHAN, J. Y BANK, R. D. (1995): Green ledgers: Case studies in corporate environmental accounting. Washington, World Resources Institute.

ENCUESTA ANUAL DE SERVICIOS (EAS) (2008): Panorámica del Transporte. Instituto Nacional de Estadística (INE).

ERIKSEN K.S. (2000): "Calculating external costs of transportation in Norway”. European Journal of Transport and Infrastructure Research, pp. 9-25.

FRIEDRICH, R. Y BICKEL, P. (2001): Environmental External Cost of Transport. Nueva York, Springer. 
GIBSON, K. C. Y MARTIN, B. A. (2004): "Demonstrating value through the use of environmental management accounting". Environmental Quality Management, Vol. 13, No.3, pp. 45.

HALLER, M., WELCH, E., LIN Y J. FULLA, S. (2007): "Economic costs and environmental impacts of alternative fuel vehicle fleets in local government: An interim assessment of a voluntary ten-year fleet conversion plan". Transportation Research Part D: Transport and Environment, Vol. 12, pp. 219-230.

HART, S.L. (1995): “A Natural-Resource-Based View of the Firm". Academy of Management Review, Vol. 20, pp. 986-1014

HART, S.L. Y AHUJA, G. (1996): "Does it Pay to be Green? An Empirical Examination of the relationship between Pollution Prevention and Firm Performance". Business Strategy and the Environment, Vol. 5, pp. 30-37

LEMP, J.D. Y KOCKELMAN, K.M. (2008): "Quantifying the external costs of vehicle use: Evidence from America's top-selling light-duty models". Transportation Research Part D: Transport and Environment, Vol. 13, pp. 491-504.

MAIBACH, M., SCHREYER, C., SUTTER, D., VAN ESSEN, H.P., BOON, B.H., SMOKERS, R., SCHROTEN, A., DOLL, C., PAWLOWSKA, B. Y BAK, M. (2007): Handbook on estimation of external cost in the transport sector: Internalisation Measures and Policies for All external Cost of Transport. CE Delft en colaboración con el Directorio General de Energía y Transporte de la Comisión Europea. Países Bajos.

MARTÍNEZ, O. (1989): Treinta años de política de transportes de la Comunidad Europea. Madrid, Centro de Publicaciones del Ministerio de Transportes, Turismo y Comunicaciones.

MAYERES, I., OCHELEN, S. Y PROOST, S. (1996): "The marginal external costs of urban transport”. Transportation Research Part D: Transport and Environment, Vol. 1, pp. 111-130.

NEHRT, C. (1998): "Maintainability of first mover advantages when environmental regulations differ between countries". Academy of Management Review, Vol. 23, No. 1, pp. 77-97

QUINET, E. (2004): "A meta-analysis of Western European external costs estimates". Transportation Research Part D: Transport and Environment, Vol. 9, pp. 465-476.

ROOME, N. (1992): "Developing environmental management strategies". Business Strategy and the Environment, Vol. 1, pp. 11-24

SÆLENSMINDE, K. (2004): "Cost-benefit analyses of walking and cycling track networks taking into account insecurity, health effects and external costs of motorized traffic". Transportation Research Part A: Policy and Practice, Vol. 38, pp. 593-606.

SAVAGE, D.E. Y JASH, C. (2004): International guidelines on environmental management accounting. Nueva York, International Federation of Accountants

SHARMA, S. Y VREDENBURG, H. (1998): "Proactive corporate environmental strategy and the development of competitively valuable organizational capabilities". Strategic Management Journal, Vol. 19, pp. 729-753

UNITED NATIONS DIVISION FOR SUSTAINABLE DEVELOPMENT (UNDSD) (2001): Environmental management accounting: Procedures and principles. Nueva York, United Nations Division for Sustainable Development

WATKISS, P. (2005): The Social Cost of Carbon Review: Methodological Approaches for Using SCC Estimates in Policy Assessment. Londres, DEFRA.

WERNERFELT, B. (1984): "A resource-based view of the firm". Strategic Management Journal, Vol. 5, No. 2, pp. 171-180 
